\title{
Correction to: End-to-end automatic differentiation of the coronavirus disease 2019 (COVID-19) from viral pneumonia based on chest CT
}

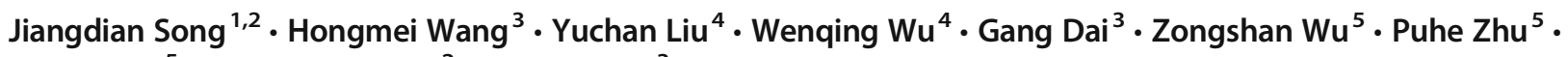 \\ Wei Zhang ${ }^{5} \cdot$ Kristen W. Yeom ${ }^{2} \cdot$ Kexue Deng $^{3}$
}

Published online: 4 March 2021

(C) Springer-Verlag GmbH Germany, part of Springer Nature 2021

\section{Correction to: Eur J Nucl Med Mol Imaging https://doi.org/10.1007/s00259-020-04929-1}

There was a mistake in the original article. One of the affiliations listed for Dr. Jiangdian Song is incorrect. The correct affiliation should have been:

${ }^{1}$ College of Medical Informatics, China Medical University, Shenyang, Liaoning, 110122, China

instead of

${ }^{1}$ Northeastern University, NO. 3-11, Wenhua Road, Heping District, Shenyang, Liaoning 110819, People's Republic of China.

The original article has been corrected.

Publisher's note Springer Nature remains neutral with regard to jurisdictional claims in published maps and institutional affiliations.

This article is part of the Topical Collection on Erratum

The online version of the original article can be found at https://doi.org/10. 1007/s00259-020-04929-1

Kexue Deng

dengkexue-anhui@163.com

1 College of Medical Informatics, China Medical University, Shenyang 110122, Liaoning, China

2 School of Medicine, Department of Radiology, Stanford University, 1201 Welch Rd, Lucas Center, Palo Alto, CA 94305, USA
3 Department of Radiology, Division of Life Sciences and Medicine, The First Affiliated Hospital of University of Science and Technology of China, No. 17, Lujiang Road, Hefei 230036, Anhui, China

4 Department of Radiology, Anhui Provincial Hospital Affiliated to Anhui Medical University, Hefei, Anhui, China

Department of Radiology, the Lu'an Affiliated Hospital, Anhui Medical University, Lu'an, Anhui, China 